\title{
Transplante de coração em pacientes com miocardiopatia chagásica
}

Adib D. JATENE*, Noedir A. G. STOLF*, Alfredo I. FIORELLI*, Edimar BOCCHI*, Lourdes HIGUCHI*, José Otávio C. AULER JÚNIOR*, Daví E. UIP*, Vicente AMATO NETO^, Fulvio PILEGGI*

JATENE, A. D.; STOLF, N. A. G.; FIORELLI, A. I.; BOCCHI, E.; HIGUCHI, L.; AULER JÚNIOR, J. O. C.; UIP, D. E.; AMATO NETO, V.; PILEGGI, F. - Transplante de coração em pacientes com miocardiopatia chagásica. Rev. Bras. Cir. Cardiovasc., 2(1):1-6, 1987

RESUMO: Entre 27 pacientes submetidos a transplante cardiaco, no Instituto do Coração do Hospital das Clínicas da Faculdade de Medicina da Universidade de São Paulo, 4 eram portadores de cardiomiopatia chagásica. As idades situaram-se entre 28 e 43 anos. Três pacientes estavam em grupo funcional IV e 1, na classe funcional II, mas apresentando taquiarritmia ventricular refratária. A imunossupressão foi feita com ciclosporina mais corticóide, no 1: paciente, e ciclosporina, azatioprina e corticóide, nos outros três. Três pacientes tiveram episódios de rejeição, tratados com pulsoterapia. Um destes pacientes apresentou doença intestinal linfoproliferativa precoce. Os 3 pacientes tiveram reagudização da doença de Chagas no 59:, 81: e 420 ' de pós-operatório, respectivamente, caracterizada por febre, lesōes cutâneas e miocardite. O Trypanosoma cruzi foi encontrado nas biópsias de pele dos 3 pacientes e nas biópsias do miocárdio em 2 pacientes. Um dos pacientes não teve reagudização. Todos os 4 doentes foram tratados com medicação específica, bem tolerada em todos. As alteraçōes da reagudização da doença de Chagas reverteram em poucos dias. Um dos pacientes, que teve doença linfoproliferativa e cujas doses de imunossupressores foram reduzidas, faleceu no $197^{\circ}$ dia, em crise de rejeição. Os demais estão bem e em seguimento por 107,160 e 500 dias, respectivamente.

DESCRITORES: transplante cardiaco, pacientes chagásicos; miocardiopatia chagásica, transplante cardiaco.

\section{INTRODUÇÃO}

O transplante cardíaco é, hoje, um procedimento de rotina para tratamento de pacientes com insuficiência cardiaca em fase terminal. A experiência acumulada neste campo tem permitido estender esta terapia a pacientes considerados, pelos protocolos de transplantes, como contra-indicados. Estão nesta situação pacientes com idade superior a 55 anos, com infarto pulmonar e em circulação assistida $^{10,11,12 .}$

A doença de Chagas tem uma alta incidência em áreas da América do Sul, Central e México. Em um estágio crônico, pode determinar grave cardiomiopatia, em uma população relativamente jo$v^{\text {vem }}{ }^{8}$. Não existe, publicada na literatura, qualquer experiência de transplante cardíaco, neste grupo.

Trabalho realizado no Instituto do Coraçāo do Hospital das Clínicas da Faculdade de Medicina da Universidade de São Paulo. São Paulo, SP, Brasil.

Apresentado ao 14: Congresso Nacional de Cirurgia Cardiaca. Salvador, BA, 27 e 28 de março, 1987

Laureado com o "Prêmio de Cirurgia Cardiaca - 1987".

- Do Instituto do Coração do Hospital das Clínicas da Faculdade de Medicina da Universidade de Sảo Paulo.

Endereço para separatas: Adib D. Jatene. Av. Dr. Enéas de Carvalho Aguiar, n: 44, 2: andar. Conselho Diretor, 05403 São Paulo, SP, Brasil. 
No Instituto do Coração do Hospital das Clínicas da Faculdade de Medicina da Universidade de São Paulo, foram submetidos a transplante cardíaco 4 pacientes com cardiopatia chagásica, e o objetivo deste trabalho é relatar esta experiência inicial.

\section{CASUÍSTICA E MÉTODO}

Durante o período de março de 1985 a janeiro de 1987 , foram submetidos a transplante cardíaco ortotópico 27 pacientes. As indicaçôes foram miocardiopatia isquêmica em 11, miocardiopatia dilatada em 10, miocardiopatia pós parto em 1 e miocardiopatia por doença de Chagas em 4. Os dados pré-operatórios destes últimos 6 pacientes estão na Tabela 1. Três pacientes estavam em classe funcional IV (NYHA) e estiveram internados na unidade de terapia intensiva, recebendo drogas inotrópicas, para tratamento de baixo débito cardíaco, em periodos diferentes, antes do transplante cardíaco. O quarto paciente estava em classe funcional II, mas com arritmias ventriculares refratárias ao tratamento clínico e que o levavam, com freqüência crescente, a procurar serviços de emergência.

A evolução clínica pré-operatória, além dos exames habituais realizados em todos os candidatos a transplante, incluiu pesquisa para detecção do parasita no sangue e testes sorológicos especificos. Foram realizados xenodiagnóstico, pesquisa do parasito por exame direto no sangue e no creme leucocitário, cultura do sangue e inoculação em coelho. Além da reação de fixação do complemento, foi feita hemoaglutinação direta com antígenos protéicos e polissacarídeos, imunofluorescência indireta específica para IgG e IgM e reação de floculação.
Não houve particularidades, em relação à técnica operatória e ao pós-operatório imediato.

As drogas imunossupressoras utilizadas foram ciclosporina A e corticóide, no paciente n: 1, e associaçāo de ciclosporina A, azatioprina e corticóide, nos demais. O paciente n: 2 deixou de receber ciclosporina $\mathrm{A}$ quando se diagnosticou doença linfoplasmocitária.

O diagnóstico de rejeição foi realizado rotineiramente, por biópsia endomiocárdica. Protocolos especiais de correlaçāo entre os achados da biópsia do ecocardiograma e do mapeamento com galium estão em estudo.

A pesquisa para detecção do parasita no sangue e os exames sorológicos específicos foram repetidos periódicamente, no pós-operatório.

Em nenhum dos pacientes foi feita terapêutica específica, no pré-operatório.

\section{RESULTADOS}

A evolução, no período de pós-operatório imediato, foi satisfatória, tendo todos os pacientes obtido alta antes de 30 dias. Em todos eles, foi observada elevação transitória de creatinina com valores entre $1,7 \mathrm{mg} / 100 \mathrm{ml}$ (caso $\mathrm{n}: 3$ ) e $2,4 \mathrm{mg} / 100 \mathrm{ml}$ (caso n: 2). Dois pacientes (casos n: 1 e 3) tiveram hipertensão moderada. Houve, ainda, infecção em 3 casos, sendo herpes simples em 1, herpes zoster em 1 e infecção de loja de marcapasso removido após o transplante em 1. Todos com evolução favorável.

Houve um óbito (caso n: 2), no 197: dia após o transplante, causado por rejeição. Este paciente apresentou uma doença linfoproliferativa envolven-

TABELA 1

DADOS PRÉ-OPERATÓRIOS

\begin{tabular}{|c|c|c|c|c|c|}
\hline CASO & NOME & SEXO & IDADE & $\begin{array}{l}\text { CLASSE } \\
\text { FUNCIONAL } \\
\text { (NYHA) }\end{array}$ & $\begin{array}{c}\text { DATA } \\
\text { OPERAÇĀO }\end{array}$ \\
\hline 6 & O. M. U. & $M$ & 43 & IV"* & 16.09 .85 \\
\hline 12 & N. R. O. & $M$ & 28 & IV ${ }^{* *}$ & 09.04 .86 \\
\hline 15 & S. C. S. & $M$ & 31 & IV** & 22.08 .86 \\
\hline 19 & G. L. M.* & $M$ & 34 & $\|^{* * *}$ & 10.10 .86 \\
\hline
\end{tabular}

\footnotetext{
- terapêutica prévia para doença de Chagas

* choque cardiogênico

*.* arritmia refratária
} 
JATENE, A. D.: STOLF, N. A. G.: FIORELli, A. I.: BOCCHI. E.: HIGUCHI. L.; AULER JÚNIOR, J. O. C.: UIP, D. E.: AMATO NETO

V.; PILEGGI, F. - Transplante de coração em pacientes com miocardiopatia chagasica. Rev. Bras. Cir. Cardiovasc. 2(1):1-6. 1987.

TABELA 2

REAGUDIZACĀO DA DOENCA DE CHAGAS

\begin{tabular}{|c|c|c|c|c|}
\hline CASO & $\mathrm{PO}$ & $\begin{array}{l}\text { DIAGNÓSTICO } \\
\text { INICIAL }\end{array}$ & CLINICA & PATOLOGIA \\
\hline 6 & $420^{\circ}$ & Clínico & $\begin{array}{l}\text { Febre ICC } \\
\text { lesão pele }\end{array}$ & $\begin{array}{l}\text { paniculite }+T \text {. cruzzi } \\
\text { miocardite }+T \text {. cruzzi }\end{array}$ \\
\hline 12 & $81^{*}$ & Clinico & $\begin{array}{l}\text { Febre ICC } \\
\text { disfunçāo } \\
\text { ventricular } \\
\text { moderada }\end{array}$ & $\begin{array}{l}\text { paniculite }+T . \text { cruzzi } \\
\text { miocardite }\end{array}$ \\
\hline 15 & - & - & - & - \\
\hline 19 & $59^{*}$ & $\begin{array}{l}\text { Biópsia } \\
\text { endomio- } \\
\text { cárdica. }\end{array}$ & $\begin{array}{l}\text { Febre-lesão } \\
\text { de pele } \\
\text { BAV + ICC }\end{array}$ & $\begin{array}{l}\text { paniculite }+T \text {. cruzzi } \\
\text { miocardite }+T \text {. cruzzi }\end{array}$ \\
\hline
\end{tabular}

- após pulsoterapia com corticóide-benzonidazol

do grande parte do intestino delgado. Houve sangramento intestinal maciço, exigindo laparotomia de urgência e ressecção de segmento de jejuno. Em conseqüência, as drogas imunossupressoras foram diminuídas, o que facilitou novas crises de rejeição. Este paciente teve, ao todo, 8 episódios de rejeição, aos $16,31,35,120,133,151,162$ e 185 dias após o transplante.

As sucessivas crises de rejeição levaram à deterioração progressiva da função cardíaca. $O$ exame anátomo patológico não mostrou evidências de recorrência do linfoma, ou doença de Chagas em atividade.

Apenas 1 paciente (caso n: 3) não apresentou episódios de rejeição. Os outros 2 tiveram, respectivamente, dois episódios moderados, com 293 e 455 dias (caso n: 1) e um episódio no $37^{\circ}$ dia (caso n: 4) após o transplante.

As crises toram tratadas com pulsos de corticóide e, apenas no caso n: 2 , foi, também, utilizada imunoglobulina antitimocitária (ATG).

Quanto à doença de Chagas, o parasita só foi detectado no sangue de 1 paciente que estava assintomático. Nos 3 restantes, houve evidências clínicas e achados histopatológicos que caracterizaram uma nova fase aguda da doença de Chagas. A reagudização foi detectada, no caso n: 1 , aos 420 dias; no caso n: 2, aos 81 dias e, no caso n: 4 , aos 50 dias após o transplante.

O diagnóstico inicial foi suspeitado por manifestaçōes clínicas, nos casos n: 1 e 2, e por presença do parasita na biópsia endomiocárdica, no caso n: 4.
As manifestações clínicas caracterizaram-se por febre alta e lesōes cutâneas com aspecto eritematoso e infiltrativo na face anterior do tórax e membros inferiores. Sinais de disfunção ventricular acompanhada de insuficiência cardiaca ocorreram nos casos no 1 e 2. Bloqueio atrioventricular e disfunção ventricular leve, diagnosticados ao ecocardiograma, foram verificados no caso n: 4 .

O estudo histológico nas biópsias da pele, realizados nos 3 pacientes, mostrou uma paniculite envolvendo o subcutâneo, com edema, focos de necrose, infiltrado celular com predomínio de macrófagos, linfócitos, plasmócitos e neutrófilos (Figura 1). O Trypanosoma cruzi foi identificado facilmente e em grande quantidade, nos pacientes $n$ : 1 e n: 4 e detectado com dificuldade, no paciente n. 2. A natureza do parasita foi confirmada usando-se técnica de imunoperoxidose.

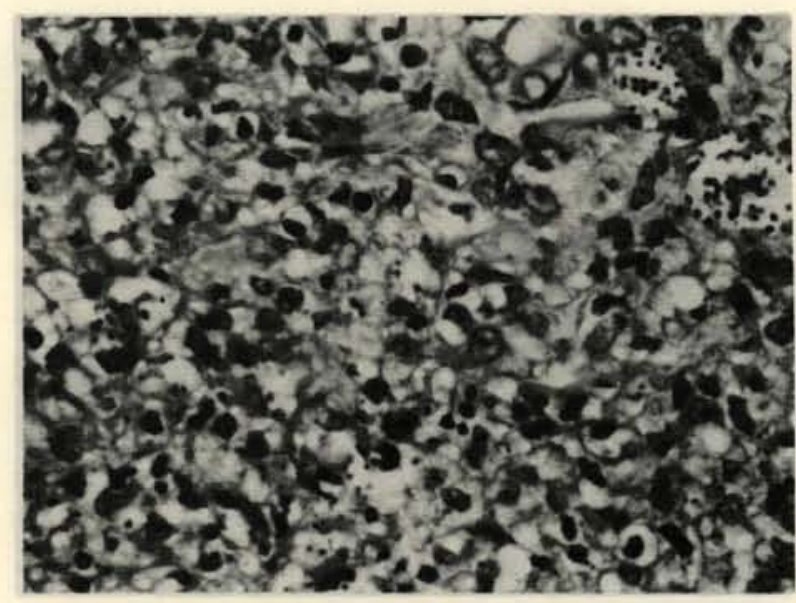

Fig. 1 - Tecido subcutâneo obtido por biópsia (paciente n: 1), mostrando infiltrado de células plasmáticas, linfócitos, macrófagos. Nestas últimas células existem formas amastigotas de tripanosoma. 
JATENE, A. D.: STOLF, N. A. G.: FIORELli, A. I.: BOCCHI, E.; HIGUCHI, L.; AULER JÚNIOR, J. O. C.: UIP. D. E: AMATO NETO. V.; PILEGGI, F. - Transplante de coração em pacientes com miocardiopatia chagásica. Rev. Bras. Cir. Cardiovasc. 2(1):1-6, 1987.

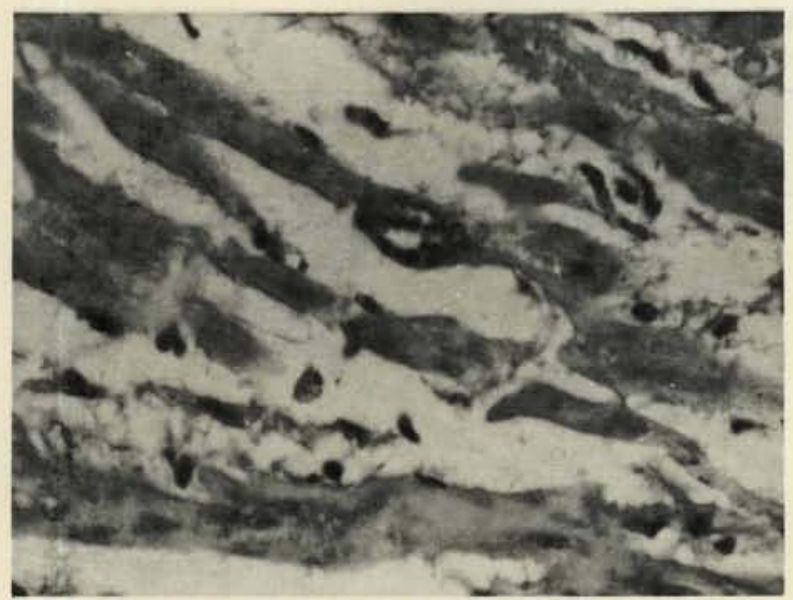

Fig. 2 - Miocárdio obtido por biópsia endomiocárdica (paciente n: 1). mostrando formas amastigotas de tripanosoma dentro das células

Nas biópsias endomiocárdicas, havia evidências de miocardite crônica com infiltração de linfócitos. Em 2 pacientes, (casos n: 1 e 4) havia, também, nas fibras miocárdicas, formas amastigotas de Trypanosoma e, no paciente n: 2 , não foi encontrado o parasita.

A pesquisa direta do parasita e os testes sorológicos falharam, na deteç̧ão da nova fase aguda.

Os 3 pacientes foram tratados com benzonidazol, na dose de $10 \mathrm{mg}$ por quilo de peso, durante 60 dias. As lesões da pele e a febre desapareceram entre 2 e 4 dias, com normalização progressiva da função cardíaca. Não houve qualquer reação adversa ao benzonidazol.

As biópsias ıniocárdicas sucessivas revelaram resolução progressiva do processo inflamatório e o parasita não foi mais detectado (Figura 3).

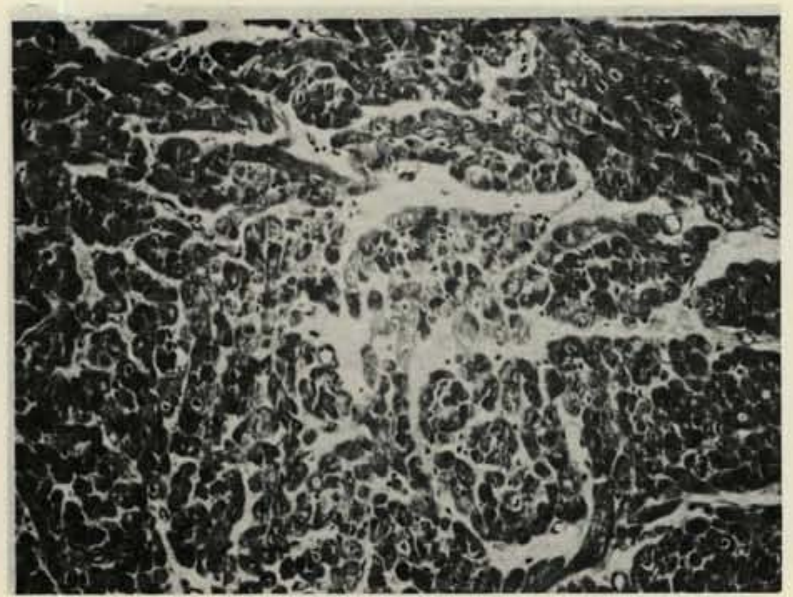

Fig. 3-Miocardio com aspecto normal obtido pós biópsia endomiocárdica (paciente $n: 1$ ) duas semanas após tratamento específico para doença de Chagas.
Os 3 pacientes que sobreviveram estão com seguimento de 500 dias (caso n: 1), 160 dias (caso n: 3) e 107 dias (caso n: 4), todos em classe funcional I pela classificação da NYHA. Dois pacientes voltaram às suas atividades profissionais.

\section{DISCUSSĀO}

A doença de Chagas apresenta uma alta incidência nas Américas Central e do Sul, estendendo-se até o México. Alguns casos foram relacionados no Texas. Estima-se em cerca de 10 a 13 milhões as pessoas acometidas por este mal, na América Latina.

O reservatório natural deste parasita são animais silvestres e a transmissão é feita por um inseto do gênero triatomídeo, popularmente conhecido como "barbeiro", que transporta o parasita no seu intestino. O parasita pode penetrar, através da mucosa intacta, ou pela pele, no local da picada para sugar o sangue e, nesta ocasião, a forma tripomastigota do parasita penetra e multiplica-se no local, dentro das células, como forma intracelular chamada amastigota. Posteriormente, as células se rompem, liberando o parasita na corrente sangüínea, quando, então, a infecção dissemina-se. Outras formas de transmissão estão documentadas e, dentre elas, a mais importante é através de transfusões sangüíneas.

$\mathrm{Na}$ fase aguda, o tripanosoma é facilmente detectado no sangue. No estágio crônico, a resposta imunitária torna difícil a sua detecção. Vários mecanismos podem levar os pacientes a desenvolverem afecções digestivas, como megacolo, megaesôfago e/ou cardiomiopatia ${ }^{3,8}$.

A indicação do transplante cardíaco em pacientes portadores de doença de Chagas originou uma argumentação fundamental, que é o comportamento do paciente chagásico crônico, submetido a imunossupressão. Informaçōes clínicas de alguns pacientes com doença de Chagas, submetidos a transplante de rim, mostraram que estes pacientes apresentaram boa evolução ${ }^{2}$.

Quanto aos aspectos experimentais, os dados disponíveis, até o momento, em relação à ciclosporina $^{4,5}$, são controversos, principalmente pela particularidade na infecção chagásica em animais de diferentes espécies ${ }^{6,7}{ }^{7}{ }^{9}$. RASSI*, utilizando corticóides em portadores de pênfigo foliácio, observou

- A. Rassi, Comunicação pessoal, 1987 
aumento da parasitemia que tinha relação com a dose empregada.

Levando em conta estes aspectos e, principalmente, considerando a má condição clínica dos receptores humanos candidatos a transplante cardíaco e os efeitos colaterais adversos das drogas empregadas para tratamento da doença de Chagas ${ }^{1}$, decidiu-se não tratar, rotineiramente, estes pacientes, quer seja no pré ou no pós-operatório, exercendo, porém, uma rigorosa vigilância clínica e laboratorial. Na hipótese de identificação de aparecimento ou aumento da parasitemia, o tratamento específico seria instituído em condições clínicas bastante melhoradas pelo transplante.

Nenhum protocolo especial de imunossupressão foi empregado, neste grupo de pacientes. No primeiro paciente (caso n: 1), foram utilizados ciclosporina e esteróides e, nos últimos 3 pacientes, uma tríplice associação de drogas (azatioprina, ciclosporina e esteróides).

As complicações observadas foram as usuais e já comentadas anteriormente, excetuando-se a proliferação linfomatosa observada no trato intestinal de um dos pacientes.

$\mathrm{O}$ aspecto mais importante relacionava-se ao comportamento da doença de Chagas frente à imunossupressão, indispensável à manutenção destes pacientes, em toda a sua evolução pós-operatória. A possibilidade de reativação da doença de Chagas, com sintomas da fase aguda e aumento da parasitemia e do parasitismo, confirmou-se. Três pacientes apresentaram quadro febril com grave comprometimento do estado geral, sem hepatomegalia, esplenomegalia e infartamento ganglionar. Chamou a atenção o fato de não se detectar o parasita no sangue, apesar de todos os exames programados terem sido realizados. $O$ diagnóstico foi feito pelo encontro do parasita em biópsia de pele, retirada de lesões cutâneas que apareceram com o quadro febril, e em biópsia endomiocárdica.

Estes 3 pacientes que apresentaram quadro de reagudização da doença de Chagas tinham sido submetidos a pulsoterapia, para controle de episódios de rejeição. O outro paciente, que não apresentou episódio de rejeição e que, portanto, não fez pulsoterapia com corticóide, não teve reagudização. Foi detectado o parasita no sangue e, por isso, recebeu, como os demais, medicação espe- cífica. Chamou a atenção o fato de, apesar da dose de $10 \mathrm{mg} / \mathrm{Hg} /$ de peso durante 60 dias, não haver qualquer reação colateral. Este fato sugere que a tolerância à medicação específica é melhor no doente imunodeprimido.

Diante desta experiência, é legítima a atitude de tratar a doença de Chagas antes do transplante cardíaco. Na hipótese de as condiçōes do paciente não permitirem o tratamento prévio, ele deverá ser administrado após a operação.

A terapêutica específica contra o parasita, em uma fase de reagudização, mostrou-se altamente eficaz, com reversão, inclusive, do quadro histológico e dos sintomas clínicos, aparentemente sem seqüelas.

\section{CONCLUSÕES}

1) O transplante de coração, em pacientes com doença crônica de Chagas, apresenta problemas especiais.

2) Pulsos de corticóides predispõem à "reagudização" da doença.

3) O quadro clínico da "reagudização" da doença de Chagas, nestes pacientes, é clinicamente diferente da fase aguda da evolução natural da doença.

4) Os exames de laboratório, para este tipo de "reagudização" da doença de Chagas, não seguem os padrões habituais.

5) O diagnóstico tem sido feito pelo quadro clínico e pelo estudo histológico de biópsias cutâneas e endomiocárdicas.

6) A terapêutica específica para doença de Chagas reverte, em poucos dias, todas as manifestações da reagudização da doença.

7) Embora maior vigilância deva ser observada, pois complicações específicas podem ocorrer, verifica-se que o transplante cardiaco pode ser realizado com bom resultado, nos pacientes com cardiomiopatia chagásica. 
JATENE, A. D.: STOLF, N. A. G.: FIORELLI, A. I.; BOCCHI. E: HIGUCHI. L.: AULER JUNIOR, J. O. C.: UIP. D. E: AMATO NETO. V.: PILEGGI, F. - Transplante de coração em pacientes com miocardiopatia chagasica. . Bev. Bras. Cir. Cardiovasc. 2(1):1-6. 1987.

RBCCV

JATENE, A. D.: STOLF, N. A. G.; FIORELLI, A. I.: BOCCHI. E.: HIGUCHI. L.: AULER JÜNIOR. J. O. C.; UIP, D. E.; AMATO NETO, V.; PILEGGI, F. - Heart transplantation in patients with chagasic cardiomiopathy. Rev. Bras. Cir. Cardiovasc., 2(1):1-6, 1987

ABSTRACT: Among 27 heart transplanted patients, 4 had chagasic cardiomiopathy. They were male. with ages from 28 to 43 years, 3 in the NYHA class IV and 1 in class II, but with refractory arrhythmias. The immunosuppressive protocol included cyclosporin and steroids in the first patient and cyclosporin, azathioprine and steroids in the last. Three patients had one or more rejection episodes and 1 had no episode. Usual complications were observed and in 1 patient a lymphoproliferative intestinal disease occurred. Three patients had a new acute phase of Chagas' disease in the 59th, 81 rst and 420 th postoperative days with fever, skin lesions and myocarditis. Trypanosoma cruzi were found in skin lesion in all 3 patients and in myocardial byopsies in 2 patients. The laboratory tests were not the expected for the acute phase. All the alterations were reversed by specific therapy in a few days. One patient died in the 197 th postoperative day due to rejection; the immunosuppression regimen had been diminished due to the lymphoproliferative disease; the others were well 107, 160 and 500 days, respectively, after the transplant.

DESCRIPTORS: heart transplantation, chagasic patients; chagasic cardiomiopathy, heart transplantation.

\section{REFERÊNCIAS BIBLIOGRÁFICAS}

1 BOAINAIN, E. - Tratamento etiológico da doença de Chagas na fase crônica. Rev. Goiana Med., 25 (1 e 2): $1-60,1979$.

2. CHOCAIR, P. R.; AMATO NETO, V.; SABBAGA, E.; TORRECILLAS, P. H. - Aspectos clínico-diagnósticos relativos à fase aguda da doença de Chagas, em pacientes submetidos a transplante de rim e imunodeprimidos. Rev. Soc. Bras. Med. Trop., 18 (1): 43-45, 1985.

3. HUDSON, L. - Trypanosoma cruzi: the immunological consequences of infection. J. Cellular Biochem., 21 (4): 299-304, 1983.

4. KIERSZENBAUM, F.; GOTTLIEP, C. A.; BUDZKO, D. B. - Exacerbation of Trypanosoma cruzi infection in mice treated with the immunoregulatory agent cyclosporin A. Trop. Med. Parasitol., 34 (1): 4-6, 1983.

5. McCABE, R. E.; REMINGTON, J. S.; ARAUjO, F. G. In vivo and in vitro effects of cyclosporin A on Trypanosoma cruzi. Am. J. Trop. Med. Hyg., 34 (5): 861-865, 1985.

6. OKUMURA, M. - Doença de Chagas experimental. In: RAIA, A. A. Manifestaçōes digestivas da moléstia de Chagas. São Paulo, Sarvier, 1983. p. 35-59.
7. OKUMURA, M.; BRITO, T.; SILVA, L. H. P.; SILVA, A. C.; CORREA NETTO, A. - The pathology of experimental Chagas disease in mice. 1. Digestive tract changes, with a reference to necrotizing arteritis. Rev. Inst., Med. Trop. S. Paulo, 2 (1): 17-28, 1960.

8. OKUMURA, M. \& CORREA NETTO. A. - Patogenia da doença de Chagas. Rev. Goiana Med., 28 (1 e 2): 77-79, 1982.

9. OKUMURA, M. \& DÉCOURT, L. V. - Estudo de efeitos da administração de drogas imunodepressoras sobre a moléstia de Chagas experimental. Rev. Hosp. Clin. Fac. Med. S. Paulo, 24 (6): 335-342, 1969.

10. SOLIS, E. \& KAYE, M. P. - The registry of the Internacional Society for Heart Transplantation: third official report. J. Heart Transplant., 5 (1): 2-5, 1986.

11. VAUGHN, C. C.; COPELAND, J. G.; CHENG, K.; AUSTIN, J.; LEVINSON, M.; EMERY, R. W. - Interim cardiac replacement with a mechanical heart: staged cardiac transplantation. Tex. Heart Inst. J., 13 (1): 45-52. 1986.

12. YOUNG, J. N.; YASBECK, J.; SPOSITO, G.; MANKAD, P.; TOWNSEND, E.; YACOUB, M. - The influence of acute preoperative pulmonary infarction on the results of heart transplantation. J. Heart Transplant, 5 (1): 20-22, 1986. 